\title{
Recherches sur la production céramique à Pompéi : l'atelier des lampes à huile (Reg. I, Ins. 20, 2-3)
}

Campagne de fouilles 2016

Laetitia Cavassa, Bastien Lemaire, Antoine Boisson, Guilhem Chapelin, Giulia Ciucci, Christine Durand, Jean-Marie Gassend, Giovanni Festa, Marcella Leone, Agnès Oboussier, Fabrice Paul, Marie Pawlowicz, Rémi Rêve, Carla Rosa, Giuseppina Stelo et Lorenzo Vollaro

\section{(2) OpenEdition} Journals

Édition électronique

URL : http://journals.openedition.org/cefr/1761

DOI : $10.4000 /$ cefr. 1761

ISSN : 2282-5703

Éditeur

École française de Rome

Référence électronique

Laetitia Cavassa, Bastien Lemaire, Antoine Boisson, Guilhem Chapelin, Giulia Ciucci, Christine Durand, Jean-Marie Gassend, Giovanni Festa, Marcella Leone, Agnès Oboussier, Fabrice Paul, Marie Pawlowicz, Rémi Rêve, Carla Rosa, Giuseppina Stelo et Lorenzo Vollaro, « Recherches sur la production céramique à Pompéi : I'atelier des lampes à huile (Reg. I, Ins. 20, 2-3) », Chronique des activités archéologiques de l'École française de Rome [En ligne], Les cités vésuviennes, mis en ligne le 21 juillet 2017, consulté le 11 septembre 2019. URL : http://journals.openedition.org/cefr/1761 ; DOI : 10.4000/cefr. 1761

Ce document a été généré automatiquement le 11 septembre 2019

(c) École française de Rome 


\title{
Recherches sur la production céramique à Pompéi : l'atelier des lampes à huile (Reg. I, Ins. 20, 2-3)
}

Campagne de fouilles 2016

\author{
Laetitia Cavassa, Bastien Lemaire, Antoine Boisson, Guilhem Chapelin, \\ Giulia Ciucci, Christine Durand, Jean-Marie Gassend, Giovanni Festa, \\ Marcella Leone, Agnès Oboussier, Fabrice Paul, Marie Pawlowicz, Rémi \\ Rêve, Carla Rosa, Giuseppina Stelo et Lorenzo Vollaro
}

\footnotetext{
Cette première campagne de fouilles de l'atelier de potier situé en Reg. I, ins. 20, 2-3 à Pompéi a eu lieu entre le 12 et le 30 septembre 2016. Il s'agit ici d'une nouvelle opération faisant partie d'un programme sur l'artisanat céramique à Pompéi, faisant suite aux précédentes missions (2012 à 2015) qui étaient alors engagées sur l'atelier de potiers de Porta Ercolano ${ }^{1}$. Cette nouvelle fouille fait partie du programme de recherches du Centre Jean Bérard (USR 3133, CNRS-École française de Rome) développé il y a un peu plus de 10 ans par Jean-Pierre Brun (Collège de France) et intitulé "Artisanat et économie à Pompéi ». Il s'agit ici d'une recherche concentrée sur l'artisanat céramique à Pompéi qui prend en considération les deux ateliers de potiers en fonction en 79 de notre ère actuellement connus. Le premier, se trouve en zone périurbaine de la ville antique, tandis que le second (dont il est question ici) se trouve en ville en Reg. I, ins. 20, 2-3 (fig. 1).
} 


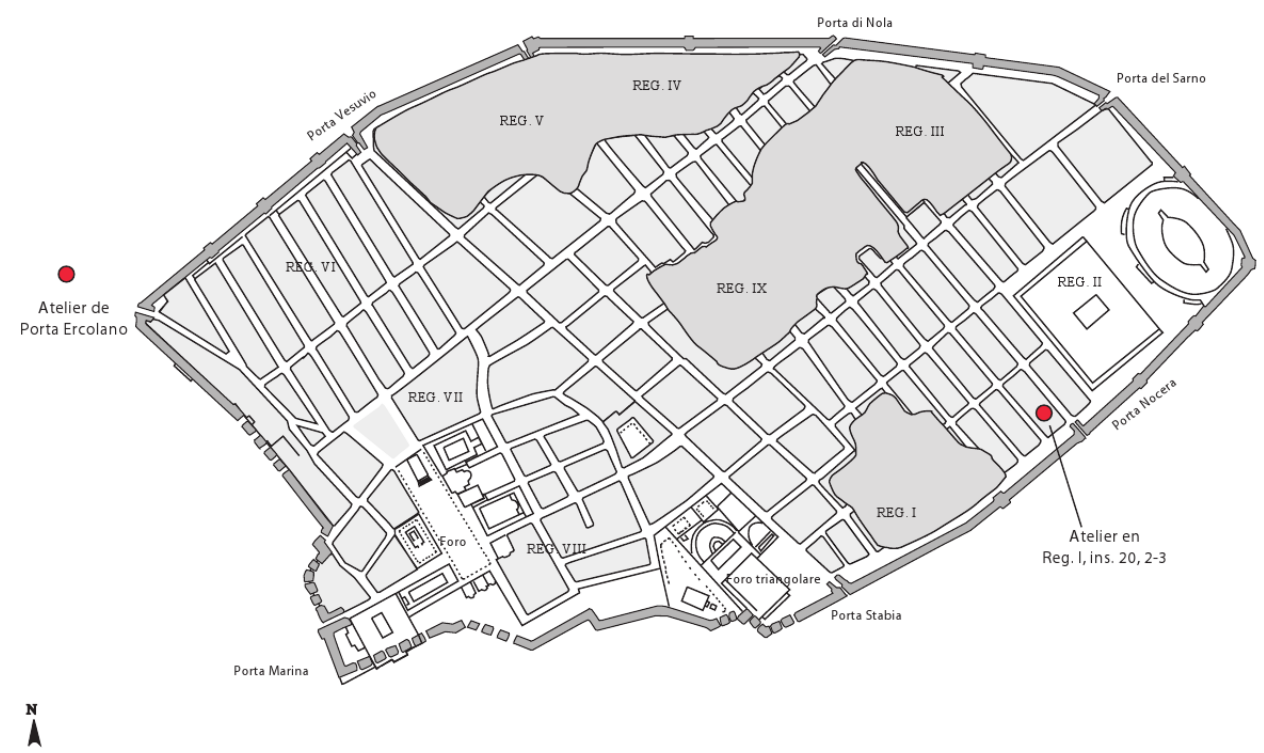

2 Ces recherches se déroulent dans le cadre d'une concession de fouilles délivrée par le Ministero per i Beni e le attività culturali, et en collaboration avec le Parc Archéologique Archéologique de Pompéi. Nous remercions le Directeur Général, Massimo Osanna, la directrice du site Grete Stefani, les fonctionnaires de la Région I, Patrizia Tabone et Marialaura Iadanza, ainsi que D. Busiello et U. Franco, responsables du dépôt de matériel.

Cette opération est réalisée grâce aux fonds du Ministère de l'Europe et des Affaires Etrangères dans le cadre de la Mission Archéologique «Italie du Sud », par l'intermédiaire du Centre Jean Bérard (CNRS USR3133-École française de Rome), ainsi que grâce au Centre Camille Jullian (Aix Marseille Université, CNRS, Ministère de la Culture et de la Communication) et au Fonds de dotation « Archéologie et Patrimoine en Méditerranée ».

\section{L'atelier des lampes à huile}

4 Cet atelier se situe au sud-est de la ville, à quelques mètres de la porte de Nocera en région I, insula 20, 2-3. Il est composé de 4 pièces et occupe une surface d'environ $110 \mathrm{~m}^{2}$ . De cet atelier, on accédait également à un jardin, appelé caupona del Gladiatore, composé d'un triclinium d'été, occupé par des vignes et dans lequel on fabriquait du vin (fig. 2). 
Fig. 2 - LOCALISATION DANS LA RÉgION I ET PLAN de L'ATELIER DES LAMPES À HUILES (PLAN G. CHAPELIN, EFR-CJB-CNRS).

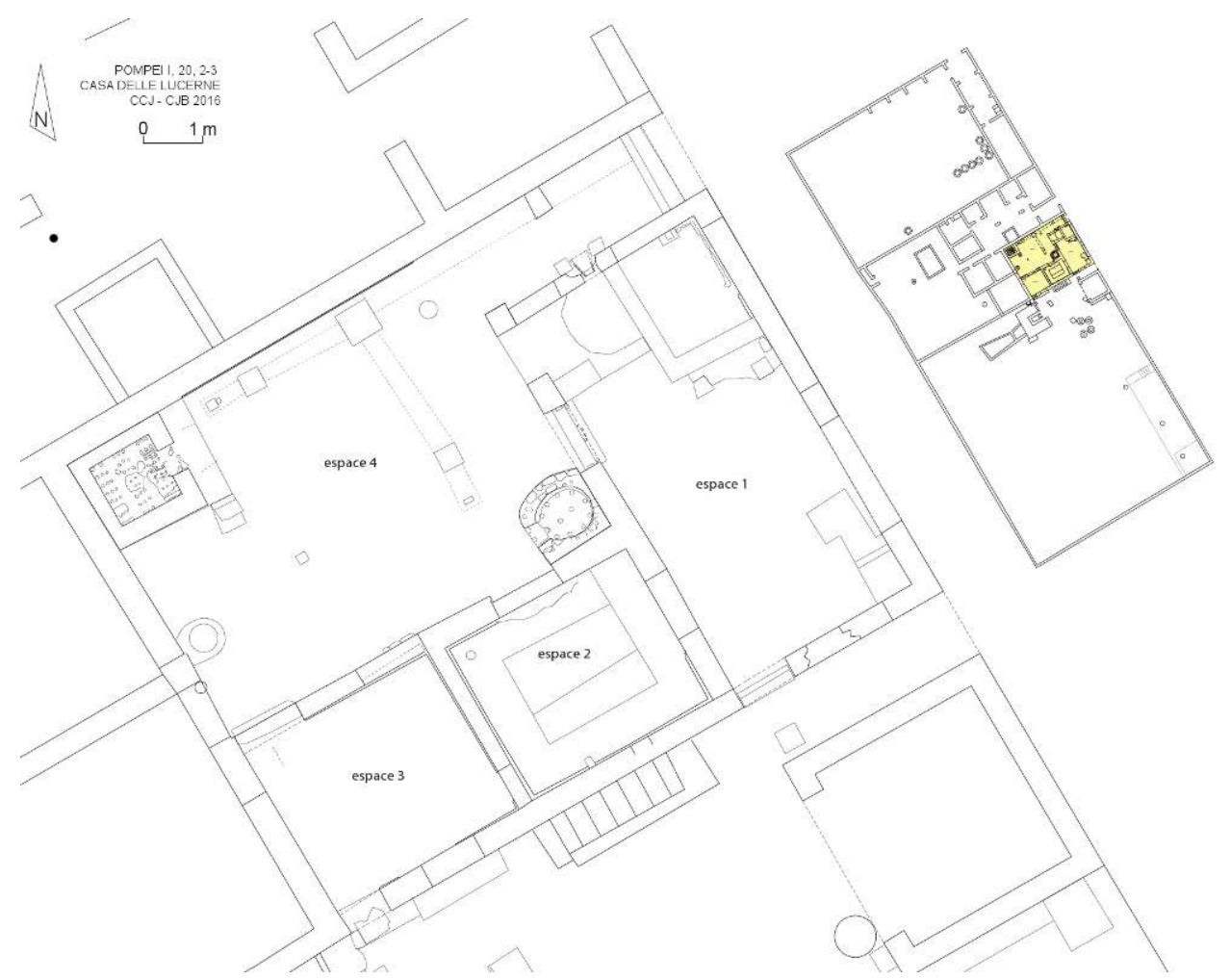

L'édifice a été dégagé entre fin 1958 et le premier trimestre 1959 sous la direction d'A. Maiuri. En 1973, Giuseppina Cerulli Irelli réalise quelques sondages bien ciblés (latrine, fosse septique...) dont elle publie les résultats, ainsi que le matériel mis au jour lors des dégagements, dans le seul article de synthèse existant à l'heure actuelle sur l'atelier. Elle publie ainsi les 61 lampes et 24 moules découverts en 1959 et mentionne également la découverte de 121 fritilli $^{2}$, petits vases réalisés au tour dont la fonction exacte demeure imprécise.

Les objectifs pour cette première campagne étaient nombreux : dégager les niveaux de 79 dans l'ensemble de l'atelier, étudier les structures présentes, et essayer d'identifier la répartition fonctionnelle de chaque pièce... avec en parallèle une étude du matériel mis au jour lors des dégagements de la fin des années 1950.

Les résultats ont été très satisfaisants : l'ensemble de l'atelier a été fouillé, un plan précis et une étude du bâti ont été mis en place.L'ensemble des structures ont été photographiées et une couverture photogrammétrique de l'atelier a été réalisée ${ }^{3}$. Nous présenterons dans ce premier article, les données relatives à la dernière phase antique, celle de 79 de notre ère concernant l'activité céramique.

\section{L'espace 1}

Cet espace mesure 3,43 $\mathrm{m}$ de large d'est en ouest pour 7,11 $\mathrm{m}$ de long du nord au sud. On y accédait par le sud-est depuis la porte $\mathrm{n}^{\circ} 2$, ouvrant sur la via Nocera. L'espace est composé de plusieurs structures bâties (fig. 3). Au sud-est, se trouve un comptoir de vente, fortement restauré, donnant directement sur la rue. Il mesure 1,95 $\mathrm{m}$ de long pour une largeur maximale de 0,90 m. Il est, à l'heure actuelle, impossible de déterminer ce qu'on y 
vendait. La production de l'atelier de potier? le vin produit dans le jardin, en vente au détail?

Fig. 3 - ESPACE 1 AVEC LE BASSIN, LA FOSSE SEPTIQUe ET LE COMPTOIR DE VENTE (CLICHÉ B. LEMAIRE, ASM, UNIV. PAUL-VALÉRY MONTPELLIER / COLLÈgE DE FRANCE-PARIS).

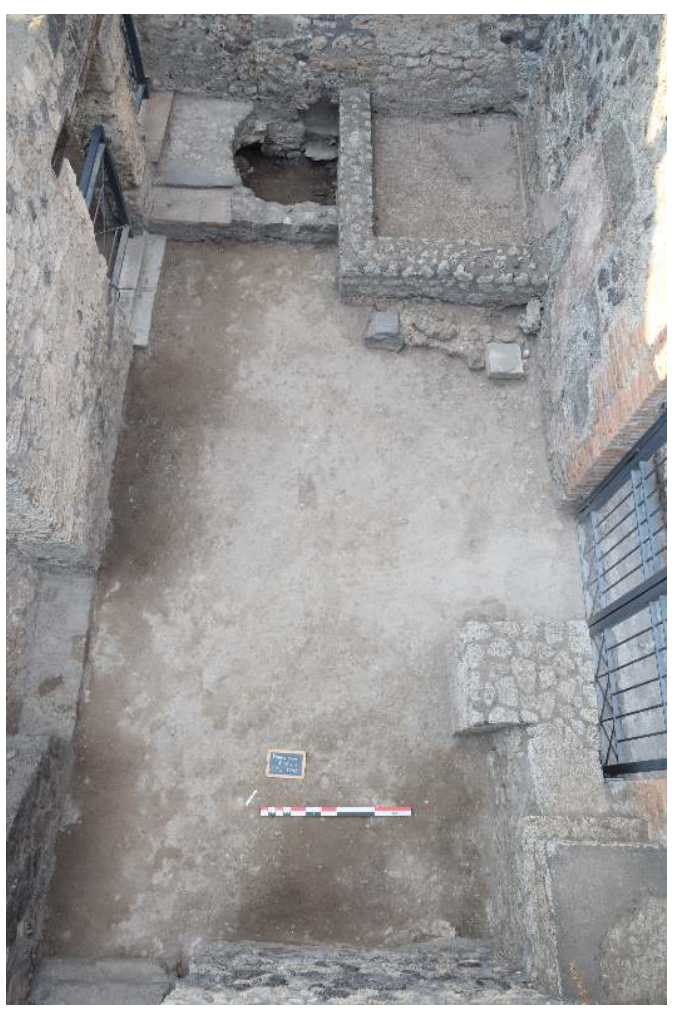

9 Au nord-est, un bassin, interprété comme bassin de décantation pour l'argile occupe l'angle de la pièce. Il a une forme quasi rectangulaire $(1,16 \mathrm{~m}$ de large pour une longueur irrégulière comprise entre 1,68 m et 1,98 m). Il est revêtu de plusieurs niveaux d'enduits en béton de tuileau, témoignages de plusieurs états.

10 Attenant au bassin, se trouve une structure, interprétée comme une fosse septique par G. Cerulli Irelli, permettant l'évacuation de la latrine située dans le couloir, au nord de la pièce 1 . De l'espace 1 , on accède à trois autres espaces : au sud le jardin ; à l'espace 2 au sud-ouest et à l'espace 4 au nord-ouest.

\section{L'espace 2}

11 Cet espace mesure 4,25 $\mathrm{m}$ d'est en ouest pour 2,25 $\mathrm{m}$ du nord au sud. On y accède par l'est par une large ouverture depuis l'espace 1. Cette pièce est circonscrite par 3 murs ayant subits plusieurs remaniements. Le mur nord a été bouché. Dans une phase précédente, la pièce était ouverte sur l'espace 4.

De même, en observant le mur sud, on observe qu'il est construit de manière assez grossière par l'emploi de toute sorte de matériau (moellons de tuf, de calcaire) mais également de céramique et l'on constate également le bouchage d'un laraire. Directement sous la terre végétale est apparu un sol de béton recouvrant l'ensemble de la pièce. Ce sol fonctionne avec deux fosses de petites dimensions (trous de poteaux ?) se trouvant dans 
l'angle nord-ouest pour l'une, et dans l'angle sud pour l'autre. La fonction de cette pièce n'est pas clairement définie.

\section{L'espace 3}

13 L'espace 3 se situe dans la partie sud-ouest de l'atelier de potier et mesure 3,52 m d'est en ouest et 3,06 $\mathrm{m}$ du nord au sud. Il est circonscrit par quatre murs et est accessible par deux portes (au nord et au sud). Aucun niveau en rapport avec la phase de 79 n'a pu être intercepté. G. Cerulli Irelli évoquant l'hypothèse, lors de ses sondages de 1973, que cette pièce n'était pas utilisée et que le sol du dernier état n'était pas présent ${ }^{4}$.

\section{L'espace 4}

L'espace 4 est la principale pièce de cet atelier, point névralgique de la production. On y accède directement de la rue par l'est par la porte 3 , à travers un couloir. Cette pièce mesure 7,25 $\mathrm{m}$ d'est en ouest pour 4,95 $\mathrm{m}$ du nord au sud, soit près de $36 \mathrm{~m}^{2}$. Elle abrite deux fours.

Cette pièce a été fortement perturbée par des travaux modernes, avec, plus particulièrement, l'installation d'un réseau de canalisations traversant l'atelier depuis la porte à l'est jusque devant le grand four à l'ouest, avec un axe nord-sud au centre de la pièce. Trois regards sont installés dans la pièce, ainsi que trois piliers soutenant les toitures de protections des fours.

Malgré cela, et malgré le fait que l'atelier ait été fouillé deux fois avant nous (dégagements en 1958 et fouilles en 1973), les vestiges antiques en place sont nombreux et viennent une fois de plus enrichir et compléter nos connaissances sur l'artisanat de la céramique à Pompéi depuis l'arrivée des matières premières jusqu'à la cuisson des vases ${ }^{5}$.

Première étape, les matières premières. Nos recherches ont permis de mettre au jour un niveau d'argile crue, de forme irrégulière mesurant $1,03 \mathrm{~m}$ de long pour $0,65 \mathrm{~m}$ de large avec une épaisseur variable entre 1,5 et $7 \mathrm{~cm}$, au pied du mur sud de la pièce. Cette découverte est de grand intérêt et pourra être comparée avec l'argile crue mise au jour dans l'atelier de Porta Ercolano. L'accès à l'eau est garanti par la présence d'un puits ou d'une citerne au pied du mur ouest.

Deuxième élément, le façonnage des vases. Comme nous l'avons vu précédemment, cet atelier est connu pour la fabrication de lampes à huile réalisées grâce à des moules ainsi que des petits vases réalisés au tour; élément dont la présence n'était jusqu'alors pas connue dans cet atelier.

Lors de la fouille de la pièce, nous avons dégagé une structure circulaire, remplie de lapilli, apparue à proximité du regard nord de l'égout moderne, au débouché du couloir, à presque $0,7 \mathrm{~m}$ au sud du mur MR6004, et 0,25m de la tranchée des égouts modernes. Il s'agit de l'emplacement du tour de potier. Sa construction est identique à ceux que nous avons mis au jour en 2014 dans l'atelier de Porta Ercolano ${ }^{6}$.

Cette structure est composée d'une panse d'amphore italique de l'aire vésuvienne, taillée et installée dans le sol antique, créant ainsi une espace vide de $28 \mathrm{~cm}$ de profondeur au centre duquel se trouve une cavité creusée à même la terre. Cette cavité est de section quadrangulaire et mesure 16 et $12 \mathrm{~cm}$ de côtés pour $30 \mathrm{~cm}$ de profondeur. Il s'agit ici du 
négatif de l'emplacement de l'axe de maintien du tour de potier. La forme de la cavité, quadrangulaire, indique clairement qu'il s'agit d'un tour à axe fixe (fig. 4).

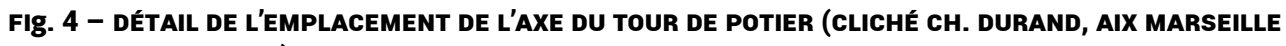
UNIV, CNRS, MCC, CCJ)

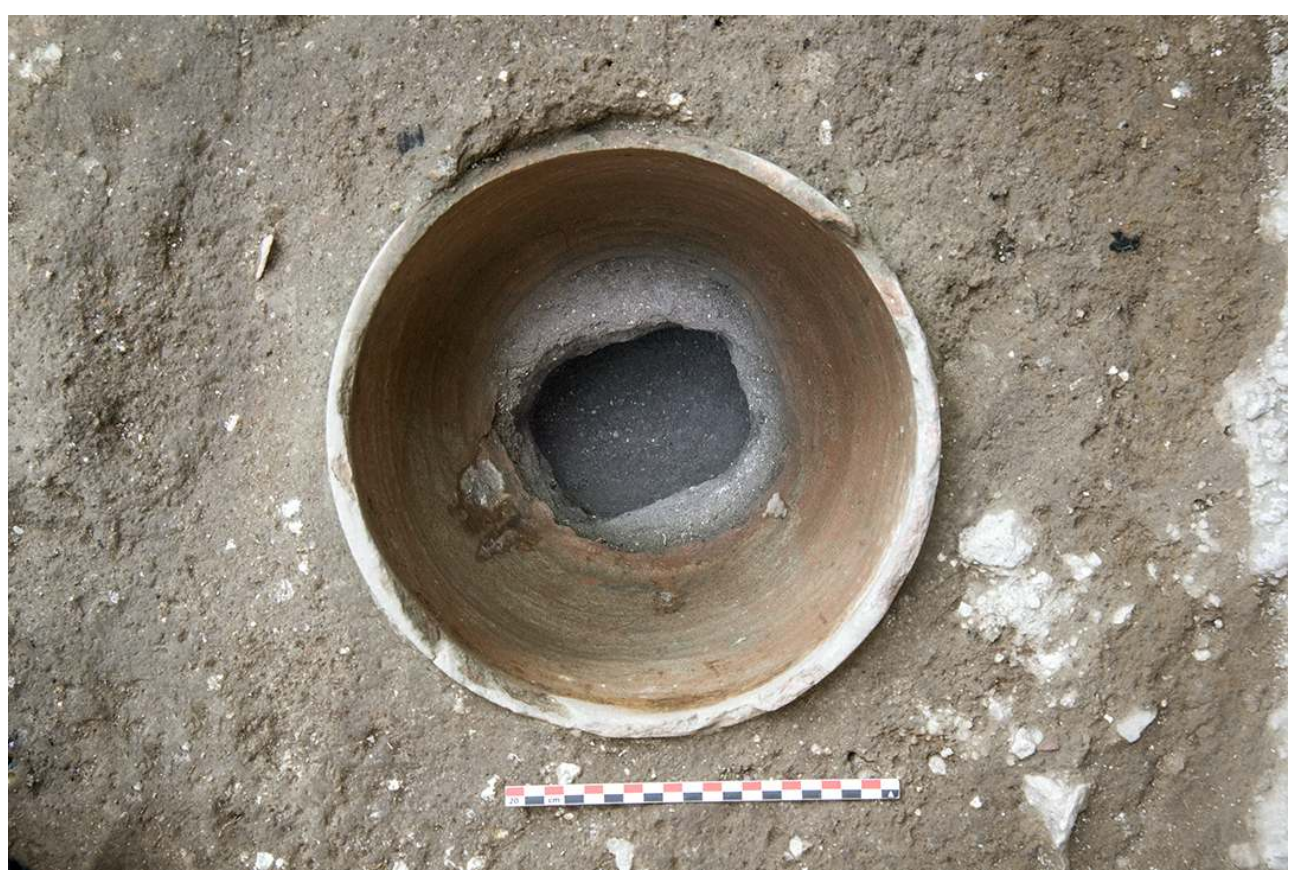

21 Le tour de potier se trouve en plein dans l'axe du couloir (long de 1,5 m pour 3,9 m de large) permettant d'accéder à la rue. L'hypothèse de G. Cirelli Irelli est que la porte d'accès était condamnée au moment de l'éruption ${ }^{7}$. Son raisonnement repose sur la présence des latrines à l'entrée même de la maison. Peut-être cela explique-t-il aussi la présence du tour de potier dans le prolongement de ce couloir?

Enfin, deux fours occupent également cet espace. Le premier, FR4002, se trouve dans l'angle nord-ouest de la pièce. Il s'agit d'un four vertical ${ }^{8}$ de forme quadrangulaire mesurant 2,09 $\mathrm{m}$ de profondeur (est-ouest) pour 1,79m de largeur (nord-sud). Il est composé de tuiles taillées, liées à l'argile et à la chaux (fig. 5). Il mesure au minimum 2,48 $\mathrm{m}$ de hauteur (dimension conservée). 
Fig. 5 - Petit four (cliché Ch. Durand, Aix Marseille Univ, CNRS, MCC, CCJ)

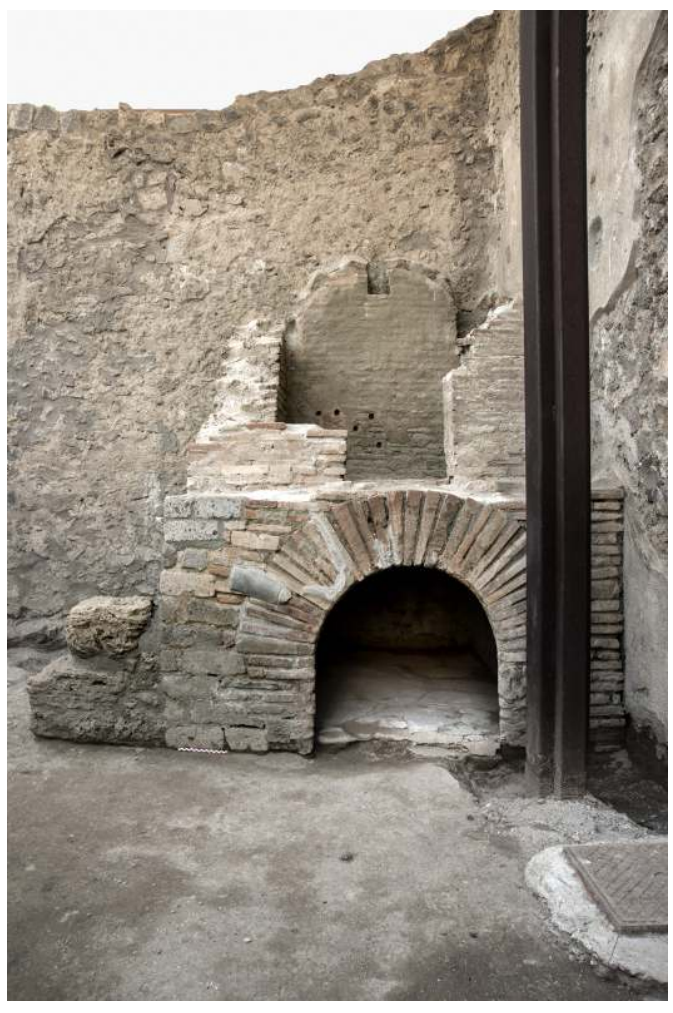

Le deuxième four est différent et est de dimensions plus modestes. Il se trouve dans l'angle sud-est de la pièce. Il s'agit d'un four circulaire à pilier central (fig.6). Il est construit en tuiles taillées. Tout comme l'autre four, une partie des murs est restaurée. 
Fig. 6 - Grand four (cliché Ch. Durand, Aix Marseille Univ, CNRS, MCC, CCJ)

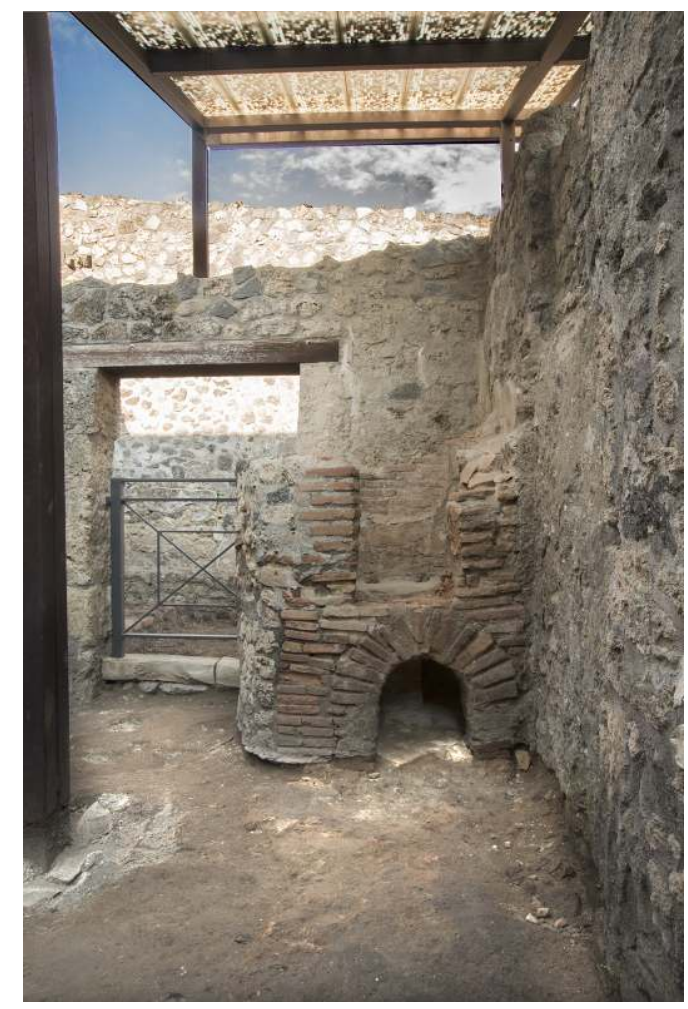

Cette première campagne de recherches dans cet atelier de potier, a permis de compléter nos connaissances dans le domaine de l'artisanat céramique à Pompéi. La découverte d'un nouveau tour et de niveau d'argile crue nous permettra de confronter les données avec l'atelier de Porta Ercolano.

\section{BIBLIOGRAPHIE}

Cavassa et al. 2012 = L. Cavassa, B. Lemaire, J.-M. Piffeteau, Pompéi, Via dei sepolcri, boutique NE, n. 29 : l'atelier de potier, dans Chronique des activités archéologiques de l'École française de Rome [En ligne], Italie du Sud, 2012, URL : http://cefr.revues.org/881.

Cavassa et al. 2013 = L. Cavassa, B. Lemaire, G. Chapelin, A. Lacombe, J.-M. Piffeteau, G. Stelo, Pompéi. L'atelier de potier de la via dei Sepolcri, 29, dans Chronique des activités archéologiques de l'École française de Rome [En ligne], Les cités vésuviennes, 2013, URL : http://cefr.revues.org/1139.

Cavassa et al. 2014 = L. Cavassa, B. Lemaire, G. Chapelin, A. Lacombe, Pompéi. L'atelier de potier de la via dei Sepolcri, 28-30, dans Chronique des activités archéologiques de l'École française de Rome [En ligne], Les cités vésuviennes, 2014, URL : http://cefr.revues.org/1279.

Cerulli Irelli 1977 = G. Cerulli Irelli, Officina di lucerne fittili a Pompei, dans L'instrumentum domesticum di Ercolano e Pompei nella prima età imperiale, Quaderni di cultura materiale, 1, Rome, 1977, p. 53-72. 


\section{NOTES}

1. Programme mené sous la direction de Sandra Zanella (Labex Archimede - Université Montpellier III, UMR 5140 Archéologie des Sociétés Méditerranéennes / UMR 8546 AOROC), Laetitia Cavassa (Centre Camille Jullian, UMR 7299, CNRS, Aix Marseille Université), Nicolas Laubry (Université Paris-Est Créteil, CRHEC - EA 4392) et Nicolas Monteix (Université de Rouen, GRHis - EA 3831) et intitulé «Organisation, gestion et transformations d'une zone suburbaine : le secteur de la Porte d'Herculanum à Pompéi, entre espace funéraire et commercial ». Pour plus de détails, nous renvoyons aux chroniques : Cavassa et al. 2012 ; Cavassa et al. 2013 ; Cavassa et al. 2014.

2. Cerulli Irelli 1977.

3. Fabrice Paul (Edikom) et Giulia Ciucci.

4. Cerulli Irelli 1977, p. 54.

5. Ces résultats sont à rapprocher des vestiges mis au jour dans l'atelier de Porta Ercolano dans lequel une grande partie de la chaîne opératoire a pu être reconstituée.

6. Cavassa et al. 2014.

7. Cerulli Irelli 1977, p. 54.

8. Par opposition à certains types de fours pour lesquels l'aire de chauffe et une partie de la structure sont semi-enterrés.

\section{INDEX}

Index géographique : Pompéi

institutions Centre Camille Jullian (Aix Marseille Université, CNRS, Ministère de la Culture et de la Communication), Centre Jean Bérard (USR 3133 CNRS/EFR), Ministère de l'Europe et des Affaires étrangères (Paris), Parco Archeologico Pompei.

\section{AUTEURS}

\section{LAETITIA CAVASSA}

Aix Marseille Univ., CNRS, MCC, CCJ-PSL (research university) EPHE

\section{BASTIEN LEMAIRE}

ASM-Archéologie des Sociétés Méditerranéennes, UMR5140, Univ Paul-Valéry Montpellier, CNRS, MCC / Collège de France-Paris

\section{ANTOINE BOISSON}

Université Paul Valéry III, Montpellier

\section{GUILHEM CHAPELIN}

Centre Jean Bérard, CNRS USR3133-École française de Rome 


\section{GIULIA CIUCCI}

IRAA, Aix Marseille Université / Università l'Orientale di Napoli

\section{CHRISTINE DURAND}

Photographe, Aix Marseille Univ, CNRS, MCC, CCJ

JEAN-MARIE GASSEND

IRAA, Aix Marseille Université

GIOVANNI FESTA

Archéologue

\section{MARCELLA LEONE}

Centre Jean Bérard, CNRS USR3133 - École française de Rome

AGNÈS OBOUSSIER

Aix Marseille Université, CNRS, MCC, CCJ

FABRICE PAUL

Edikom

\section{MARIE PAWLOWICZ}

Étudiante, Aix Marseille Université

RÉMI RÊVE

Étudiant, Aix Marseille Université

\section{CARLA ROSA}

Étudiante à l'Université de Fisciano-Salerno

GIUSEPPINA STELO

Centre Jean Bérard, CNRS USR3133 - École française de Rome

LORENZO VOLLARO

Archéologue 